\title{
Exploring Potential Usability Gaps when Switching Mobile Phones: An Empirical Study
}

\author{
Aiko Fallas Yamashita ${ }^{1,3}$ \\ Software Engineering ${ }^{1}$ \\ IT University of Gothenburg \\ SE-417 56 Gothenburg \\ +46 - 76 - 2778571 \\ aiko@ituniv.se
}

\author{
Wolmet Barendregt ${ }^{2,3}$ \\ School of Business and Informatics ${ }^{2}$ \\ University College of Borås \\ SE-501 90 Borås \\ +46 - 322639933 \\ wolmetb@hotmail.com
}

\author{
Morten Fjeld ${ }^{3}$ \\ t2i Lab (www.t2i.se), CSE ${ }^{3}$ \\ Chalmers University of Technology \\ SE-412 96 Gothenburg \\ +46 - 31 - 7721027 \\ morten@fjeld.ch
}

\begin{abstract}
The present study explores potential usability gaps when users switch from a familiar to an unfamiliar mobile phone interface. A within-subject experiment was performed in which nine users familiar with Sony-Ericsson T630 and nine familiar with Nokia 7250 performed tasks on both phones. On average, test subjects spent more time on finishing tasks with an unfamiliar phone than with a familiar one. For two of the four tasks, there was a significant difference in completion time between the first-time Nokia users and the first-time Sony-Ericsson users. The tasks of adding a contact to the address book and sending an SMS to a contact in the address book were performed more quickly by new Nokia users than by new Sony-Ericsson users. The subjective difficulty ranking also showed that first-time Nokia users found the new phone easier to use than first-time SonyEricsson users did. Hierarchical Task Analysis is used as a potential explanation, and three other theories that relate to these findings are presented: mental models, habit errors, and emotional attachment.
\end{abstract}

\section{Categories and Subject Descriptors}

H.5.2 [Information Interfaces and Presentation (e.g., HCI)]: User Interfaces - evaluation/ methodology, interaction styles (e.g., commands, menus, forms, direct manipulation), usercentred design.

\section{General Terms}

Measurement, Design, Experimentation, Human Factors.

\section{Keywords}

Mobile phone UI, usability evaluation, usability problems.

\section{INTRODUCTION}

Users of the Windows operating system are accustomed to accessing the 'Start' menu in order to shut down the computer, even though it sounds contradictory, and may even seek an analogue menu option when facing a new operating system. This is an example of what Zapf [26] calls habit error, which refers to how our perception of a system can be affected by our

(C) Aiko Fallas Yamashita, Wolmet Barendregt, Morten Fjeld, 2007 Published by the British Computer Society

People and Computers XXI-HCI... but not as we know it: Proceedings of HCI 2007

Linden J. Ball, M. Angela Sasse, Corina Sas, Thomas C. Ormerod, Alan Dix, Peter Bagnall, and Tom McEwan (Editors) habits and experience with previous systems.

According to von Niman et al. [17] "mobile phones get more and more advanced and many users of mobile services experience serious difficulties trying to set up, configure and access data services like e-mail, Internet, or messaging through their mobile devices. Users lack the expertise necessary to configure and set up their devices, services and applications appropriately. Furthermore, even the configuration of device properties to the desired behavior is often beyond the users' abilities". Mobile phone user interfaces (UIs) have different ways of organising functionalities as well as diverse interface and interaction styles [9]. The wide selection of mobile phones can even cause users to experience new difficulties when switching from one mobile interface to another. For instance, many users complain about different menus and keys with apparently 'similar' functionalities. Despite the fact that there are extensive works evaluating mobile phone usability, little research has focused on usability issues related to switching phones. We conjecture that there are usability gaps [2] in going from being an expert user of one mobile phone model to becoming a new user of another one. This other model may or may not be another brand, which is an issue we do not explicitly address here. So, our driving question is: how difficult is it to become accustomed to a new mobile phone user interface?

The study presented was carried out to shed light on what happens when users accustomed to one mobile phone switch to a new one with different interfaces and menus. For this experiment we chose the Sony-Ericsson T630 and the Nokia 7250 as testing phones, since they constitute two popular brands in the market and differ in both interaction style and user interface (see [9] for a concrete definition of interaction style). In this paper, a user's familiar mobile phone model will be referred to as the source, while the model the user is switching to will be referred to as the target. Our study addresses the following four research questions:

RQ1. Is there a difference in task-completion time between experienced Nokia and Sony-Ericsson users? (Comparison of the sources).

RQ2. Is there a difference in task-completion time between first-time Nokia and Sony-Ericsson users? (Comparison of the targets).

RQ3. When users switch from one phone to another, do they perform worse with the new phone (the target) than with their familiar phone (the source)?

RQ4. In terms of subjective assessment, is there a difference between switching from Nokia to Sony-Ericsson and vice-versa (from source 1 to target 2 and from source 2 to target 1 )? 
The research presented here may support other studies that analyse the relationship between a user's knowledge of a familiar interface and the usability of a new phone design. This may benefit mobile phone designers aiming to create more intuitive, universal, and easy-to-learn interfaces to attract users, regardless of their mobile phone background.

The next section offers a theoretical background by introducing mental models, describing related work, and presenting possible usability measurement criteria. Section 3 describes the experiment and the applied methodology. The results of the experiment are reported in Section 4. Section 5 offers a discussion along the lines of mental models, habit errors, and emotional attachment. Section 6 draws conclusions from this study while Section 7 discusses limitations and future work.

\section{THEORETICAL BACKGROUND}

First, we introduce some theory related to mental models, as it can partially explain why we should pay attention to users' mobile phone backgrounds. Then, we describe the theoretical underpinning of our experimental work, partly found in previous studies of mobile phone usability and partly in theories on how to measure such usability.

\subsection{Mental Models and Mobile Phones}

From an HCI point-of-view, a mental model is a user's set of beliefs about how an interactive system works [18]. Mental models are also related to how people organise and chunk information, something strongly related to mobile phone menu hierarchies and navigation styles [13]. An inaccurate mental model of what is happening in a system leads to errors [3]. According to Holtzblatt, a "user's familiarity with other applications influences users' mental model and expectations for mobile applications" [7].

Mental models have already been considered by HCI researchers as an important way to understand the usability of mobile devices. Kiljander [9] refers to Koivunen et al.'s [11] description of a mismatch between terminology and grouping of user interface objects with the users' mental model as one of the common usability defects in smart products. Kiljander also cites Ruuska-Kalliokulju et al. [21], which affirms that users may have inaccurate mental models of a phone interface that frequently switches modes and utilises telecommunications or computing jargon in its feedback messages.

\subsection{Mobile Phone Usability}

Several studies analyse usability requirements for mobile phones through usability evaluations and other analyses. Terrenghi et al. [24] present an overview of the current challenges for user-centred designs of mobile phones and propose a 'Framework for Usability Requirements Specification' in mobile contexts. Kiljander [9] describes contemporary mobile user interfaces and interaction styles, and reports on an experiment conducted to investigate measurable differences in usability depending on the interaction styles. It compares several different styles with the 'Three-soft' key interaction style of Nokia, thus is focusing mainly on an ' $\mathrm{n}: 1$ ' (many to one) comparison in contrast the presented crosscomparison. Ziefle [27] studies usability issues with reference to mobile phones' complexity levels and the user's expertise level. Soriano et al. [23] present SMS tests performed with middle aged users, implying the need to segment users in order to attain more accurate results of users' needs. Park et al. [19] analyse how a user's trust or affection for a brand affects usability tests. Lee et al. [12] present a detailed description and a case study of a systematic usability evaluation process (SEMCPU) based on a methodological triangulation using five empirical methods: scenario-based task performance, questionnaires, retrospective think-aloud, post-task interviews, and user observation. Kaikkonen et al. [8] explore the suitability of laboratory experiments as opposed to field testing for usability evaluation. Klockar et al. [10] detail a controlled experiment for analysing usability aspects of different mobile phones. Klockar et al. use keystrokes as quantitative data, analysing them in order to find possible enhancements for mobile interfaces, but without performing any specific statistical analysis.

\subsection{Usability and Measurement Criteria}

According to Fu Qiu et al. [25], who cite Shackel [22], the usability of a system can be defined as "the capability in human functional terms to be used easily and effectively by the specified range of users, given specified training and user support, to fulfil the specified range of tasks, with the specified range of environmental scenarios". Some references in Terrenghi et al. [24] include properties such as effectiveness, learnability, flexibility, and user satisfaction. In other studies, properties such as efficiency, ease of memorisation, and low error rate are mentioned as usability indicators [15]. Fu Qiu et al. perform a usability test of a mobile phone camera by establishing four usability dimensions: perception, control/action, learning/memorisation, and evaluative feeling. These seem to better suit consumer electronic products (a more detailed description can be found in $[5,24])$. Although Fu Qiu et al.'s model is better suited for smart devices, it is quite complex, requiring a great number of measurements and considerable effort in terms of experimental design and setup. For our study, we will measure the following properties because they are well known and commonly suggested measurements for evaluating overall usability of systems or devices (also encompassed in the Fu Qiu et al. model) [4]:

M1. Effectiveness (the level of completeness with which users achieve the goals).

M2. Efficiency (the resources needed to achieve the goals; in this case, time).

M3. User satisfaction (the comfort and subjective acceptance of the user to the product).

Finally, some other works [16] include these three usability measurement criteria, and provide a process for defining mobile usage scenarios and mapping them to a set of guidelines in order to improve accessibility to mobile services.

\section{EXPERIMENTAL DESIGN}

In order to address the four research questions (RQ1-RQ4), an evaluation was performed in which users had to switch from their familiar mobile phone to an unfamiliar one. A questionnaire was then used to measure the users' satisfaction level. In this section, the experimental design is described.

\subsection{Participants}

An experiment with eighteen participants was carried out. Nine of the test subjects were experienced Sony-Ericsson users; nine were experienced Nokia users. None of the test subjects had any previous experience with the target phone or similar ones. The test subjects had, on average, seven years of experience using mobile phones, and most of them were either engineering students or university graduates. 


\subsection{Experimental Variables}

There were two independent variables, the source and the target mobile phone, each with the two levels: Sony-Ericsson and Nokia.

There were two dependent variables: (i) successful completion of task (yes or no); and (ii) task completion time (in seconds).

\subsection{Experimental Procedure}

A within-subject experiment was performed in a semicontrolled environment (a room in the university with a moderate level of noise), where test subjects were instructed to perform certain tasks using two different mobile phones: a familiar type and an unfamiliar type. Potential learning-effects from the task repetition were balanced out by using a LatinSquare design [14]. The results of the experiment were registered by the experimental leader using paper and pencil protocol. Test subjects were given ten minutes to finish each of the four tasks they were given. The experimental tasks were as follows:

\section{T1. Add a new contact to the address book.}

T2. Write a specific SMS message and send it to a specific contact in the address book.

T3. Make a reminder for a birthday on the calendar.

T4. Switch the profile to silent (mute).

These four tasks were chosen because they represent common tasks that can be performed on almost any modern mobile phone within a reasonably short amount of time. The tasks give a good overview of the various common functionalities of mobile phones, ranging from more simple (such as adding a new contact) to more complex ones (such as adding a birthday reminder to the calendar)

If unable to finish a task within ten minutes, test subjects were asked to proceed to the next one. After solving the task, test subjects were asked to answer three questions measuring the tasks' level of difficulty (1: easy, 2: fairly easy, 3 : somewhat difficult, 4: difficult) and one measuring readiness to switch mobile phone user interfaces. The three questions examined were:

Q1. Level of difficulty compared to their phone.

Q2. Level of difficulty in finding a function.

Q3. Level of difficulty in using a function.

\subsection{Equipment}

The experiment was carried out with two mobile phones, each with similar capabilities, features, and release dates: SonyEricsson T630 and Nokia 7250 (Figure 1). Detailed information regarding the two models is available on the companies' respective web sites.

\section{RESULTS}

For T4, some users had created 'fast-keys' for this function in their own phones, making results for source and target phones incomparable. Hence, we only consider results for T1, T2, and T3. For each of these three tasks we answer research question RQ1, RQ2, and RQ3. As RQ4 does not directly relate to any one distinct task, it is discussed separately.

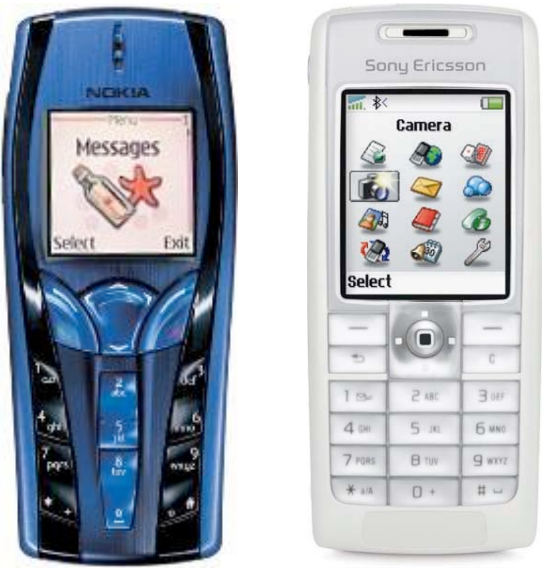

Figure 1. The two telephones used in the experiment were the Nokia 7250 (left) and the Sony-Ericsson T630 (right).

\subsection{Task 1: Adding a New Contact to the Address Book}

Figures 2 and 3 show the completion times on task one for Nokia and Sony-Ericsson users, respectively, on the source phone and the target phone. Completion times for experienced Nokia and Sony-Ericsson users were not significantly different (RQ1). This means that experienced users could perform the tasks on their source phone equally fast, independent of the model.

Completion time was significantly higher, $\mathrm{t}(16)=4.09, \mathrm{p}<0.01$ (two-tailed), for test subjects using the Sony-Ericsson phone as a target (RQ2). This means that unfamiliar users can more quickly add a new contact in the Nokia address book than in the Sony-Ericsson address book.

When users switch from one phone to another, do they perform worse with the target phone than with their source phone (RQ3)? A t-test was performed for completion times, and, not surprisingly, it took significantly more time, $\mathrm{t}(34)=-3.32, \mathrm{p}<$ 0.01 (one-tailed), for the test subjects to complete this task on the target phone than on the source phone.

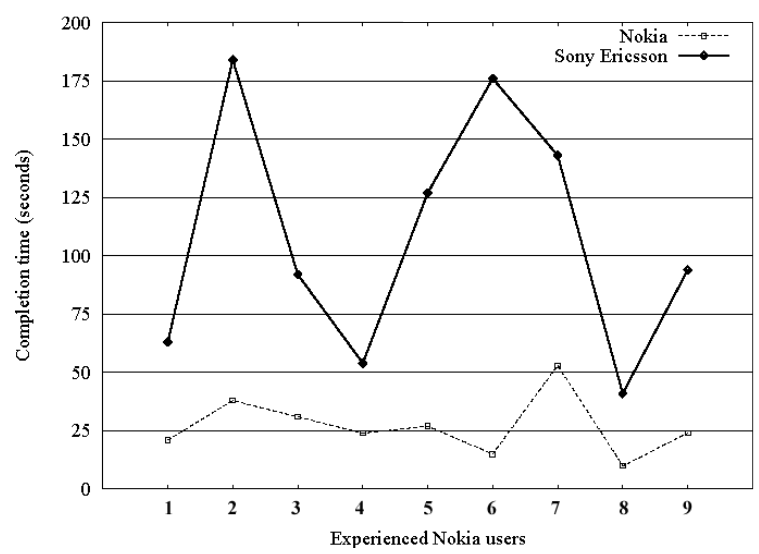

Figure 2. Time spent on task 1 by users with Nokia as their source using both a Nokia phone and a Sony-Ericsson phone. 


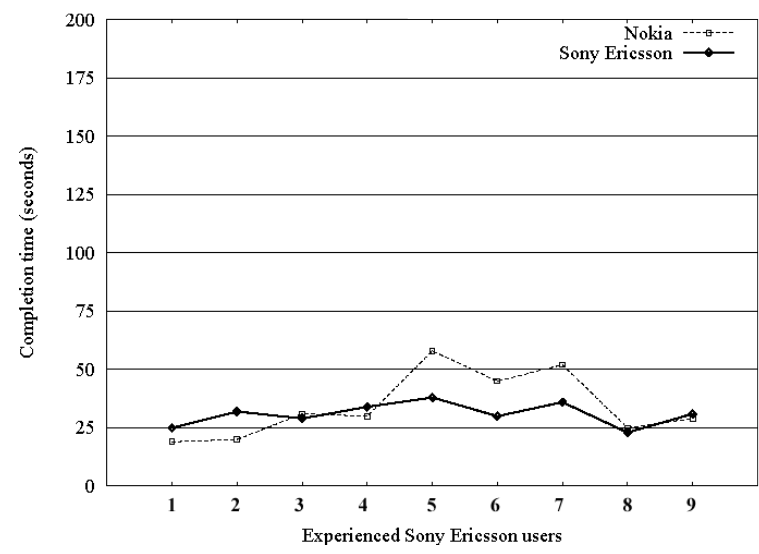

Figure 3. Time spent on task 1 by users with a SonyEricsson source using both a Sony-Ericsson phone and a Nokia phone.

\subsection{Task 2: Writing and Sending an SMS to a Contact in the Address Book}

Participant 5 in the Nokia group did not finish the task within ten minutes; therefore the maximum completion time of 600 seconds was assigned (Figure 4). The results of the SonyEricsson group are given in Figure 5.

Again, completion times for experienced Nokia and SonyEricsson users were not significantly different (RQ1).

In order to perform a t-test on the difference in performance for the second task, the data from participant 5 in the Nokia group were omitted because he did not complete the task on the target phone, and so, did not yield a valid completion time. The t-test shows that completion time was significantly higher, $\mathrm{t}(15)=$ $3.54, \mathrm{p}<0.01$ (two-tailed), for test subjects using the SonyEricsson phone as a target (RQ2). This means that unfamiliar users can more quickly create and send an SMS message to a contact in the address book using the target Nokia phone, than users with Sony-Ericsson as a target phone.

When users switch from one phone to another, do they perform worse with the target phone than with their source phone (RQ3)? A t-test was performed for completion times in this task, and again, it took significantly more time, $\mathrm{t}(32)=-3.85, \mathrm{p}$ $<0.01$ (one-tailed), for the test subjects to complete this task on the target phone than on the source phone.

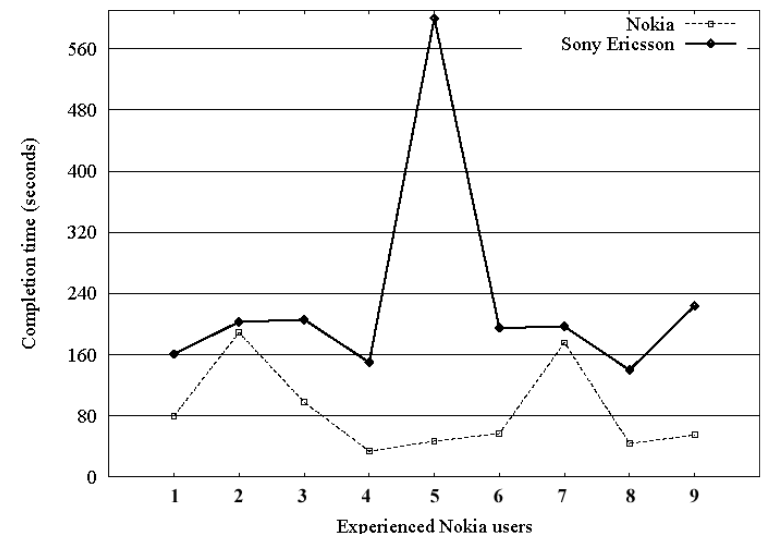

Figure 4. Time spent on task 2 by users with Nokia as their source using both a Nokia phone and a Sony-Ericsson phone.

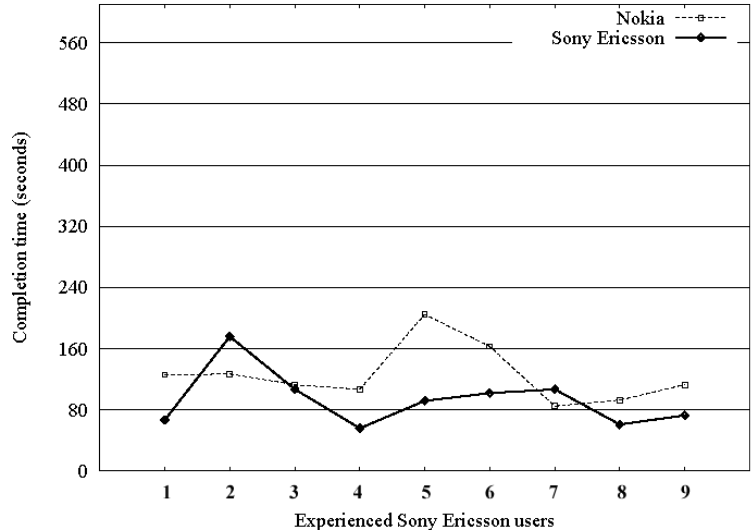

Figure 5. Time spent on task 2 by users with Sony-Ericsson as their source using both a Sony-Ericsson phone and a Nokia phone.

\subsection{Task 3: Making a Reminder for a Birthday in the Calendar}

Participant 3 in the Nokia group did not finish the task within ten minutes on their source phone; therefore the maximum completion time of 600 seconds is assigned (Figure 6). In order to perform t-tests for RQ2 and RQ3, the data of participant 3 in the Nokia group were omitted because he did not complete the task, and so did not yield a valid completion time. The results of the Sony-Ericsson group are given in Figure 7.

Again, completion times for experienced Nokia and SonyEricsson users were not significantly different (RQ1).

The t-test shows that completion time was not significantly higher for test subjects using the Sony-Ericsson phone as a target (RQ2).

When users switch from one phone to another, do they perform worse with the target phone than with their source phone (RQ3)? A t-test was performed for completion times in this task and again it took significantly more time, $\mathrm{t}(32)=-3.41, \mathrm{p}<0.01$ (one-tailed), for the test subjects to complete this task on the target phone than on the source phone.

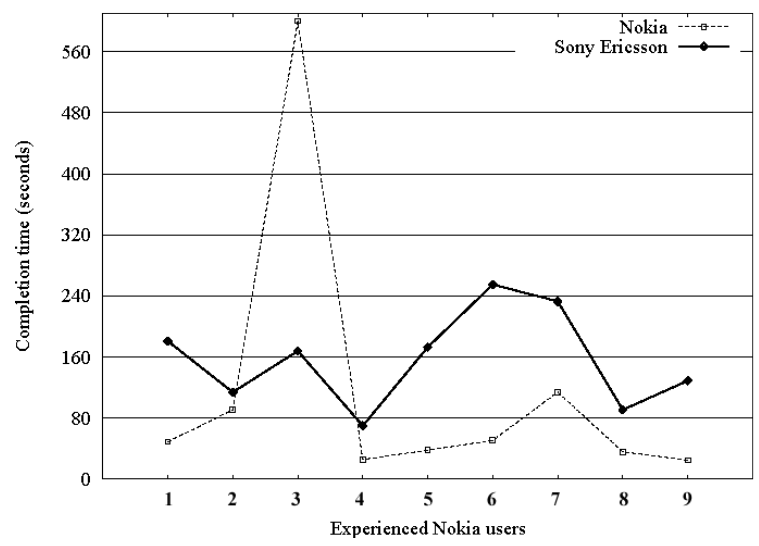

Figure 6. Time spent on task 3 by users with a Nokia source using both a Nokia phone and a Sony-Ericsson phone. 


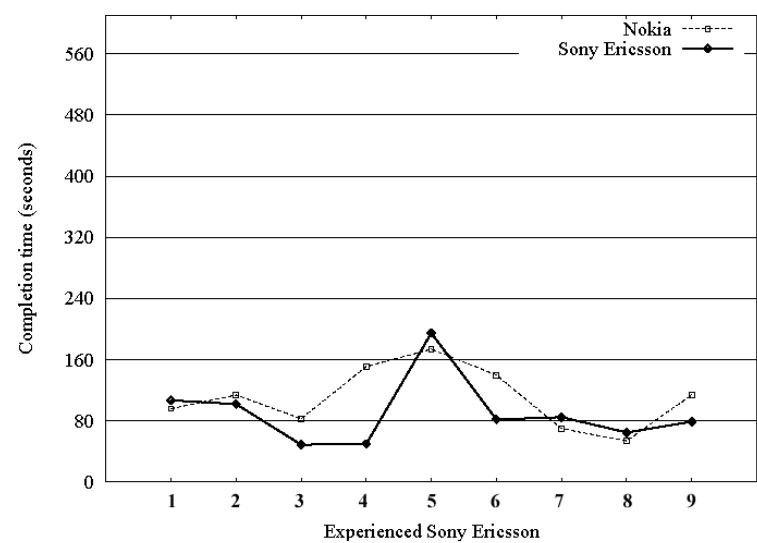

Figure 7. Time spent on task 3 by Sony-Ericsson users using a Sony-Ericsson phone and a Nokia phone.

\subsection{Subjective Results}

In terms of subjective assessment, is there a difference between switching from a Nokia to a Sony-Ericsson phone and viceversa (RQ4)? The results of the questionnaire are given below (Figures 8-10); no answer from participant 3 in the Nokia group. In each of the Figures it is clear that the experienced Sony-Ericsson users find it easier to use the Nokia phone than vice-versa. This is in congruence with the objective results measured in terms of completion times. Finally, some additional comments are presented from both testing groups (Figures 11-12).

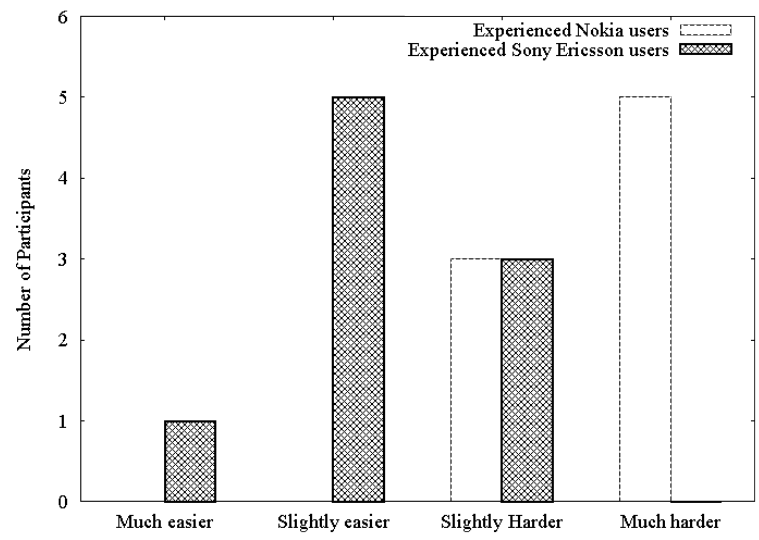

Figure 8. Question one: General difficulty in using the target phone, grouped by source phone.

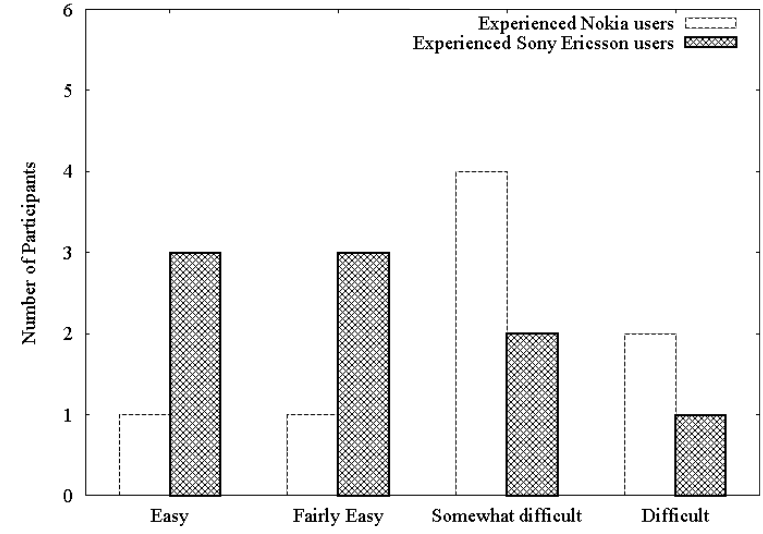

Figure 9. Question two: Level of difficulty in finding required functionality in target phone, grouped by source phone.

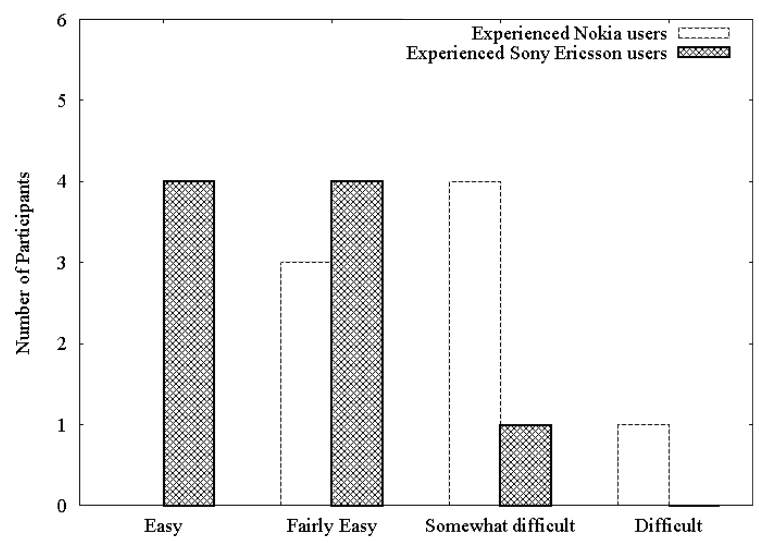

Figure 10. Question three: Level of difficulty in using required functionality in target phone, grouped by source phone.

\section{"...Not very intuitive menus, more interaction would be nice..." \\ “...I don't like the way in which you access the functions with the Sony Ericsson, I had problems navigating with it..."}

"Overall I liked the Nokia better but that is because I've owned one for a long time..."

Figure 11. Comments on Sony-Ericsson phones from users with Nokia as source.

"There are too much graphics and is slow in both phones. It is very annoying with Nokia with the full screen pictures of each menu option"

"Special characters in Nokia are very difficult to type"

"The calendar is better in Nokia; all other is as good in both phones"

"My phone has prettier icons..."

Figure 12. Comments on Nokia phones from users with Sony Ericsson as source.

\subsection{Task Analysis: HTA}

A significant result is that Sony-Ericsson users found it less difficult to switch to a Nokia phone than vice-versa. This is apparent from the differences in completion time and the subjective difficulty ranking. To explain these findings, we considered cognitive modelling of mobile phone interaction. One approach is the extended Keystroke-Level Model (KLM) [6]. Also, expert mobile phone menu interaction was modelled using GOMS and ACT-R [1]. For our work, in which developing an exact model was not the goal, we chose to employ Hierarchical Task Analysis (HTA) [3]. We employed HTA on both phones in T1, where first-time Nokia users performed better than first-time Sony-Ericsson users (RQ2). The HTA of Sony-Ericsson showed that the task is slightly more complex with this phone than with a Nokia phone, because it requires additional steps in order to accomplish the task. These additional steps were required in order to select the type of phone number (e.g., office number, home number, 
mobile number). The user was also forced to use the navigation functions while inserting the contact information. For instance, in Sony-Ericsson phones, after the user has inserted the contact name, they must select the information to be inserted next (i.e., phone number, email, etc.), whereas the Nokia phone automatically requested the next required data (in this case, the phone number).

Both the results of the experiment and the HTA can be related to Ziefle [27] who affirms that "systems built with a rather complex interface are more difficult to use and provoke additional and misleading user actions that are not helpful for completing a specific phone function". In the experiment performed by Ziefle, the Nokia phone was less complex than the other phones tested, resulting in higher efficiency measurements. An HTA for adding a contact with the Nokia phone used in Ziefle's experiment was carried out and compared to our Nokia phone, resulting in the same level of complexity for performing the task. This suggests that switching from a more complex phone to a simpler one is easier than vice-versa.

\section{DISCUSSION}

We conclude that is not possible to discard the existence of usability gaps when a user with previous experience is faced with a new interface. In other words, we suggest that a user cannot switch smoothly from one mobile phone to another without some difficulties or challenges.

We assume that when a user comes into contact with a new mobile phone interface (whether he/she has previous experience with other phones or not) there is a learning curve. We suggest that the learning process when switching mobile phones is different from the case when the user has no previous experience with mobile phones.

However, the results of this experiment do not address the case when a user with absolutely no mobile phone experience uses a mobile phone for the first time. In this sense, the study cannot demonstrate that it is harder for someone who has significant experience with one mobile style to learn a new interface than for someone with absolutely no experience to use an interface for the first time.

When learning a new interface, the user with previous experience might encounter problems for several reasons. A possible explanation could be that the new system is difficult to use (or more complex, as mentioned before), not very intuitive, etc. Another explanation could be the existence of discrepancies between the users' current mental model and the functionality of the new phone. This last factor can be also related to users' habit errors [26] which occur when a user has been using a certain interface for a while and incorrectly applies knowledge about the familiar interface in using the unfamiliar interface. Some other factors to consider are the user's emotional attachment to previous models or brands, their background, and which segment of the user population they belong to.

\subsection{Mental Models}

The studies briefly cited in Section 2.1 address the problem of mental models in a broader perspective, but other studies such as Ziefle [27] have determined the influence of users' experience in the usability of mobile devices. Ziefle stresses the need of determining constellations of mobile phones that may weaken or even cancel the advantage of expertise, that is, "...the experienced, sophisticated mental model of an expert does not cover a 'new' technical configuration where previous solving approaches as well as approved rules show to be insufficient..." [27]. In this case, efficiency measurements in the experiment suggest that the mental model of the experienced users may not have helped enough in order to seamlessly adapt to the new phone (or could even have negatively impacted the process). Further studies are required in order to confirm this.

An interesting comment from one of the users, concerns navigation style: “...I don't like the way in which you access the functions..." (Figure 11). This user's comment is in line with the results of the subjective assessment, since localisation and the usage of functionalities are normally associated with navigation (see Figures 8-9). Regarding this comment, Lee [12] defined three components of menu navigation-interface metaphors, mental models, and design-driven interaction. Lee suggested that a design error in any one of those components can induce errors in another one. Lee gives an interesting example related to mental models: "For example, if the menu hierarchy in the cell phone does not conform to users' mental models, they cannot perform navigation correctly". The results from this study are indicators that it may either be a design issue in the Sony Ericsson phone or the mental model of Nokia users that is not well-suited to Sony Ericsson phones. In order to answer this question, further studies examining the navigation style of Sony Ericsson phones must be performed with users without any previous mobile phone experience.

\subsection{Habit Errors}

As explained above, when people use a certain mobile phone frequently they establish a mental model of how the phone works. When switching from one phone to another they may have problems, because they have to create a new mental model of the phone, and, naturally, they may wrongly use their old mental model in a new situation. This is closely related to mismatches as defined by Zapf et al. [26], who define a taxonomy of human error types when using office applications. In particular, the errors that Zapf et al. call "habit errors" can occur when switching between navigation styles. Reason [20] also mentions strong habit intrusions as one of the causes of human error, where a frequently invoked rule is applied inappropriately.

Ziefle mentions an example in their study that can be interpreted as a habit error: “...when interfaces rely on contra intuitive learning rules that defy learnability and fool the user each time he tries again (example: the key that is misinterpreted as confirmation key due to its position and size)". In the context of this work, an example could be a key that is used for confirming an action in one phone is similar in size and shape to a key used for cancelling an action in a new phone. In such a case, users may face difficulties with the new phone due to habit errors.

Kiljander [9] states that there is no difference in switching from one interaction style to another, but does not consider elements such as habit errors. The interaction style could remain the same, but the location of the functionalities as well as the design of the hardware interface may trigger usability issues in consideration of habit errors.

In this study, a closer analysis of the occurring errors was not performed (measurement of total number of errors, among other things), so a direct correlation between habit errors and usability difficulties in this context could not be established. For this study, it was loosely assumed that the presence of errors would arise in the efficiency measurement, assuming that each time a user makes a mistake he/she needs to perform additional tasks in order to recover, resulting in an increase of completion 
time. But a strict correlation could not, by any means, be established through this study. Furthermore, an analysis on the type of observed errors would also be required.

\subsection{Emotional Attachment}

Another interesting comment from one of the users was the following: “...I have owned for a long time..." (Figure 11). This connotes a certain emotional attachment to the previous phone, which, due to the difference of brands in the tested phones, should be noted. Park et al. [19] state that subjective perception of ease-of-use of a new UI does not directly correlate to the effectiveness and efficiency. In our case, the first time Sony Ericsson users' subjective assessments regarding ease-of-use were aligned with the quantitative results. Another interpretation for this comment could be that the user is habituated to the Nokia phone style, which is indicative of relationship between the difficulty in interacting with a new phone and the duration of exposure to the old one.

This motivates us to reconsider the segment of users that have emotional attachment to brands and/or models, as well as users with mental models that have remained relatively unchanged for a long period of time. Soriano [23] offers examples of how segmentation of users may be a key factor in successful product design. However, we deem the issue of segmentation to be outside the scope of the work presented here.

\section{CONCLUSION}

Aiming to answer the research questions raised in the first section we conclude from this study that:

RQ1. There is no difference in task-completion time between experienced Nokia and Sony-Ericsson users. This means that experienced Nokia and Sony-Ericsson users can perform all of the tasks equally fast.

RQ2. There are differences in task-completion time between first-time Nokia and Sony-Ericsson users. For tasks, T1 and T2, first-time Nokia users are faster than first-time Sony-Ericsson users.

RQ3. When users switch from one phone to another they perform worse with the new phone (the target) than with their familiar phone (the source). This means that, in accordance with what users often say, it is more difficult to perform many common actions on a new phone than on a familiar phone.

RQ4. In terms of subjective assessment, there is a difference between switching from Nokia to Sony-Ericsson and vice-versa (from source 1 to target 2 and from source 2 to target 1 ). In congruence with the greater completion times for some of the tasks on the new phones, the first-time Nokia users found the unfamiliar phone easier to use than the first-time Sony-Ericsson users did.

The HTA shows that, for some tasks, the Sony-Ericsson phone is indeed slightly more complex than the Nokia phone. Since experienced Sony-Ericsson users do not have greater completion times than experienced Sony-Ericsson users, it also means that users overcome this initially hindering factor after some time.

\section{LIMITATIONS AND FUTURE WORK}

A limitation of this work is that no significant qualitative data, such as log files or verbal protocols, were gathered. This could have given more insight into why users with a Sony-Ericsson phone as their source found it easier to use a Nokia phone, rather than vice-versa. But the fact that these differences exist shows that usability issues within this context may vary depending on diverse factors, among them the nature of the source and the target interfaces, as well as the users' mobile phone background.

As mentioned before, we have not been able to establish any strict correlation between habit errors or mental models and usability problems when switching between mobile phones. These were suggested as possible answers aligned with the exploratory character of the study presented.

A future study in which more qualitative data are gathered by means of thinking-aloud evaluation may yield greater insight into the users' mental models. In particular, an analysis of specific types of user problems with a certain source phone could be useful to determine whether the mental model of one phone influences the use of another phone.

The work presented by Kiljander is a good reference that could be used for analysing different elements of a mobile device's interaction style and their impact on users with great habit intrusion.

Another suggestion for future work is the comparison of user workload (i.e., level of frustration, mental effort, etc.) in order to better analyse the learning process that the user is exposed to when switching to a new mobile phone. This type of study involving a greater number of participants would be desirable in order to produce robust statistical results.

\section{ACKNOWLEDGEMENTS}

We thank Sofia Andersson, Alexander Mansouri, and Mikael Törnqvist for their help in carrying out the experiments.

\section{REFERENCES}

[1] Amant, R. S., Horton, T. E., and Ritter, F. E. Model-based evaluation of expert cell phone menu interaction. $A C M$ Transactions on Computer-Human Interaction, 14, 1 (2007), 1-24.

[2] Bevan, N. Practical issues in usability measurement. Interactions, 13, 6 (November 2006), 42-43.

[3] Dix, A., Finlay, J., Abowd, G., and Beale, R. HumanComputer Interaction (Third Edition). Pearson/Prentice Hall, Harlow, UK, 2004.

[4] Frøkjær, E., Hertzum, M., and Hornbæk, K. Measuring usability: are effectiveness, efficiency, and satisfaction really correlated? In Proceedings of the SIGCHI conference on Human factors in computing systems (CHI 2000). ACM Press, New York, NY, 2000, 345-352.

[5] Han, S. H., Yun, M. H., Kwahk, J., and Hong, S. W. Usability of consumer electronic products. International Journal of Industrial Ergonomics, 28 (2001), 143-151.

[6] Holleis, P., Otto, F., Hussmann, H., and Schmidt, A. Keystroke-level model for advanced mobile phone interaction. In Proceedings of the SIGCHI conference on Human factors in computing systems (CHI 2007). ACM Press, New York, NY, 2007, 1505-1514.

[7] Holtzblatt, K., Ketola, P., and Turner, T. Techniques for designing mobile applications with customer data. In $\mathrm{CHI}$ 2004: Extended abstracts of the SIGCHI conference on Human factors in computing systems. ACM Press, New York, NY, 2004, 1604-1605.

[8] Kaikkonen, A., Kallio, T., Cankar, M., Kekäläinen, A., and Kankainen, A. Usability testing of mobile applications: A 
comparison between laboratory and field testing, Journal of Usability Studies, 1, 1 (2005), 4-16.

[9] Kiljander, H. Evolution and Usability of Mobile Phone Interaction. Ph.D. Dissertation, Helsinki University of Technology, 2004.

[10] Klockar, T., Carr, D. A., Hedman, A., Johansson, T., and Bengtsson, F. Usability of mobile phones. In Proceedings of the 19th international symposium on Human factors in telecommunications. (Berlin, Germany, 2003). 2003, 197 204.

[11] Koivunen, M-R., Nieminen, M., and Riihiaho, S. Usability Defects in Smart Products. TKO-B131, Helsinki University of Technology, Otaniemi, Finland, 1996.

[12] Lee, Y. S., Hong, S. W., Smith-Jackson, T. L., Nussbaum, M. A., and Tomioka, K. Systematic evaluation methodology for cell phone user interfaces. Interacting with Computers, 18, 2 (2006), 304-325.

[13] Lindholm, C. Mobile Usability: How Nokia Changed the Face of the Mobile Phone. McGraw-Hill Companies, Blacklick, OH, 2003.

[14] Mandl, R. Orthogonal Latin squares: An application of experiment design to compiler testing. Communications of the ACM, 28, 10 (1985), 1054-1058.

[15] Nielsen, J. Usability Engineering. Academic Press, San Diego, CA, 1993.

[16] von Niman, B., Böcker, M., Schneider-Hufschmidt, M., Flygt, M., Parodi, P., Ketola, P., Williams, D., and Tate, $\mathrm{M}$. Enabling and improving the use of mobile e-services. In Proceedings of Mobile HCI 2005. ACM Press, New York, NY, 2005, 357-358.

[17] von Niman, B. Böcker, M., Schneider-Hufschmidt, M., Flygt, M., Parodi, P, Ketola, P., Williams, D., and Tate, M.. Enabling and Improving the Use of Mobile e-Services, http://portal.etsi.org/stfs/STF_HomePages/STF285/STF28 5.asp

[18] Norman, D. The Design of Everyday Things. Doubleday/Currency, New York, 1988.

[19] Park, S., Harada, A., and Igarashi, H. Influences of personal preference on product usability. In CHI 2006:
Extended abstracts of the SIGCHI conference on Human factors in computing systems. ACM Press, New York, NY, 2006, 87-92.

[20] Reason, J. T. Human Error. Cambridge University Press, Cambridge, UK, 1990.

[21] Ruuska-Kalliokulju, S., Schneider-Hufschmidt, M., Väänänen-Vainio-Mattila, K., and von Niman, B. Shaping the future of mobile devices: Results of the CHI 2000 workshop on future mobile device user interfaces. SIGCHI Bulletin (January/February 2001), 16.

[22] Shackel, B. Usability: Context, framework, definition, design and evaluation. In B. Shackel and S. J. Richardson (eds.), Human factors for informatics usability. Cambridge University Press, New York, NY, 1991, 21-37.

[23] Soriano C., Raikundalia G., and Szajman J. A usability study of short message service on middle-aged users. In Proceedings of the 19th conference of the computerhuman interaction special interest group (CHISIG) of Australia on Computer-human interaction. CHISIG of Australia, Narrabundah, Australia, 2005, 1-4.

[24] Terrenghi, L., Kronen, M., and Valle, C. Usability requirements for mobile service scenarios. In Proceedings of HCI International 2005. (Las Vegas, 2005). 2005.

[25] Yuan Fu Qiu, Yoon Ping Chui, and Helander, M. G. Usability analysis of mobile phone camera software systems. In Proceedings of the 2006 IEEE conference on Cybernetics and intelligent systems. (Bangkok, June 2006). 2006, 1-6.

[26] Zapf, D., Brodbeck, F., Frese, M., Peters, H., and Prumper, J. Errors in working with office computers: A first validation of a taxonomy for observed errors in a field setting. International Journal of Human-Computer Interaction, 4 (1992), 311-339.

[27] Ziefle, M. The influence of user expertise and phone complexity on performance, ease of use and learnability of different mobile phones. Behaviour \& Information Technology, 21, 5 (2002), 303-311. 